University of Wollongong

Research Online

Faculty of Engineering and Information

Faculty of Engineering and Information

Sciences - Papers: Part A

Sciences

$1-1-2013$

\title{
A methodology for cost optimization of the layout design of multi-span reinforced concrete beams
}

\author{
Pezhman Sharafi \\ University of Wollongong, psharafi@uow.edu.au \\ Muhammad Hadi \\ University of Wollongong, mhadi@uow.edu.au
}

Lip H. Teh

University of Wollongong, Iteh@uow.edu.au

Follow this and additional works at: https://ro.uow.edu.au/eispapers

Part of the Engineering Commons, and the Science and Technology Studies Commons

Research Online is the open access institutional repository for the University of Wollongong. For further information contact the UOW Library: research-pubs@uow.edu.au 


\title{
A methodology for cost optimization of the layout design of multi-span reinforced concrete beams
}

\author{
Abstract \\ This paper presents a description of a new methodology for the optimum preliminary layout design of \\ reinforced concrete multi-span beams considering the relevant cost elements. First, a new objective \\ function is presented, as an alternative to traditional cost functions for reinforced concrete beams. The \\ ability of being easily employed in layout optimization problems, gives the new cost function a distinct \\ advantage over its alternatives. Examples are included to illustrate the performance of the new \\ methodology.

\section{Keywords} \\ layout, design, multi, span, reinforced, concrete, beams, methodology, optimization, cost

\section{Disciplines} \\ Engineering | Science and Technology Studies

\section{Publication Details} \\ Sharafi, P., Hadi, M. N. \& Teh, L. H. (2013). A methodology for cost optimization of the layout design of \\ multi-span reinforced concrete beams. Proceedings of the Fourteenth International Conference on Civil, \\ Structural And Environmental Engineering Computing (p. 124). United Kingdom: Civil-Comp Press.
}




\title{
A Methodology for Cost Optimization of the Layout Design of Multi-Span Reinforced Concrete Beams
}

\author{
P. Sharafi ${ }^{* 1}$, Muhammad N. S. Hadi ${ }^{2}$, Lip. H. Teh ${ }^{3}$ \\ School of Civil, Mining and Environmental Engineering, University of Wollongong, \\ Northfields Avenue, Wollongong, NSW 2522, Australia
}

\begin{abstract}
In many optimum design cases, we look for a set of design variables selected from a given list which assure optimum of objective function together with satisfaction of constraints. That is, optimum conceptual design mostly consists of selecting the best combination of finite number of structural elements and available parameters. In this paper a novel technique is presented for conceptual design optimization of framed buildings with rectangular plan. The method supports all the buildings with grid pattern column layout. To that end, we take advantages of knapsack problem as a basic applied combinatorial optimization problem. The objective is to find rectangular column layout of maximum profit for frames that also satisfy the imposed geometric constraints. The method is presented in a general form and is not confined to optimization under a certain type of action effect and/or geometrical constraints. It gives the methodology the ability of being formulated for various objective functions and constraints and different structural systems.
\end{abstract}

Keywords: column layout design, rectangular plan, combinatorial optimization, knapsack problems

\section{Introduction}

In the phase of buildings' conceptual design, an appropriate preliminary geometric layout of structures is of great importance, as it impacts the entire subsequent stages of the design process. In cost optimization of structures, a non-optimum preliminary

\footnotetext{
${ }^{1}$ Research Associate/PhD candidate, School of Civil, Mining and Envir. Eng., Univ. of Wollongong, Wollongong, NSW, Australia.*(Corresponding author).Email address: ps170@uow.edu.au

${ }^{2}$ Assoc. Professor, School of Civil, Mining and Envir. Eng., Univ. of Wollongong, Wollongong, NSW, Australia.

${ }^{3}$ Senior Lecturer, School of Civil, Mining and Envir. Eng., Univ. of Wollongong, Wollongong, NSW, Australia
} 
layout (conceptual) design optimization, may result in sub-optimal solutions, as they suffer from the problem of not necessarily having an optimum starting point.

The major barrier to automated preliminary design of buildings is the large size problem resulted from the vast number of design variables and constraints. When it comes to choosing an appropriate column layout among a huge number of possible dimensions, the result is to deal with a large combinatorial optimization problem. It gives highly nonlinear nature to automated preliminary design procedure, and makes the design optimization process rather cumbersome. These facts cause most studies to be confined to optimization of buildings with predetermined shapes.

With rapidly advancing computer technology, nevertheless, computers are becoming more powerful, and correspondingly, the size and the complexity of the problems that can be solved using optimization techniques are also increasing. Such considerable advances have taken place in computer methods can be of substantial aid in the creative process of designing the best system, and enables designers to evaluate more alternatives. On the other hand, progress in modern metaheuristics enables the designers to deal with large size problems.

In the most optimum design cases, we look for a set of design variables selected from a given list which assures optimum objective function together with satisfaction of constraints. That is, the optimum design consists of selecting the best combination of finite number of structural elements and available parameters. Such combinatorial nature, from the mathematical point of view, gives the optimum design procedure a discrete nature.

Combinatorial optimization, arising in various applications in engineering and management, is one of the most active fields in the interface of computer science, applied mathematics and engineering. It has the advantage that thousands of real-life problems can be formulated as combinatorial optimization problems. Furthermore, historically, many of the strongest and most frequently used algorithms in combinatorial optimization are based on the connection between geometry and optimization. It gives such methods the ability of being employed for solving the geometry and topology related optimization problems due to ease of formulation [13].

This paper presents a combinatorial optimization approach for preliminary layout optimization of framed buildings of rectangular patterns. The number and size of spans in both orthogonal directions can be variables. The objective is to find layouts of maximum profit for frames with rectangular plans that satisfy the imposed dimensional, strength and serviceability constraints. The term profit, depending on the problem's objective function, can be interpreted as minimum cost, minimum lateral displacement, maximum energy saving or any other objective. The method, which takes advantage of an applied combinatorial optimization problem, namely the knapsack problem, is presented in a general form and is not confined to optimization under a certain type of action effect and/or geometrical constraints.

\section{Knapsack Problems}


As a basic combinatorial optimization, knapsack problems arise when we want to select an optimum subset of bounded weight from a set of items each of which has a weight and a profit. Each item consumes a known amount of resources and contributes a known benefit. Items are to be selected in a way which maximizes the total benefit without exceeding a given amount of resources [4]. In knapsack problems, it is typically assumed that the profits and weights are positive.

Given a set of $n$ items and a set of $m$ knapsacks with limited capacity of $a_{j}$ each $(m \leq n)$, the multiple knapsack problem is the problem of selecting $m$ subset of items so that the total profit of the selected items is a maximum. Formally

$$
\begin{gathered}
\max f=\sum_{\mathrm{j}=1}^{\mathrm{m}} \sum_{\mathrm{i}=1}^{\mathrm{n}} b_{i j} y_{i j} \\
\sum_{\mathrm{i}=1}^{\mathrm{n}} r_{i j} y_{i j} \leq a_{j} \quad j \in\{1,2, \ldots, m\} \\
y_{i j}=\left\{\begin{array}{l}
1 \\
\text { if Item } i \text { is chosen for knapsack } j
\end{array}\right. \\
\text { if is not chosen for knapsack } j
\end{gathered}
$$

where $b_{i j}$ and $r_{i j}$ respectively indicate the profit and the weight of Item $i \in$ $I=\{1,2, \ldots, n\}$ when selected for Knapsack $j$.

\section{Statement of the Problem}

A challenging problem facing designers in the preliminary layout design phase is how to efficiently represent the column layouts. An ideal system for the preliminary layout design of buildings is one which results in an economic design and in parallel, fully considers all aspects of the process and the imposed constraints. To achieve such an ideal system, one will require major advances in multi-objective decision. Therefore, a practical preliminary layout design, in addition to satisfying the architectural constraints must consider the achieved profit as well $[5,6]$.

In order to describe the column layouts of buildings of rectangular floor plan, it is practical to divide the floor plan by smaller rectangular areas. That is, a column layout can be described as an arrangement of rectangular areas that completely fill the entire rectangular floor area. In other words, a column layout can be created by subdividing the entire floor area into several rectangular areas with no gaps as shown in Fig. 1. Any variation in the building's column layout is equivalent to the variation of rectangles' dimensions. Various arrangements of any set of rectangular subareas that carpet the floor plan, and satisfy the geometric constraints are feasible solutions. Each feasible solution represents both a column layout design and a rectilinear pattern for floor plan. Therefore, selecting the optimum set of rectangles and their optimum arrangement among the sets of feasible solutions results in an 
optimum layout design. The objective is a pattern for floor plan including the columns layout design, which provides the maximum profit for the preliminary design phase.

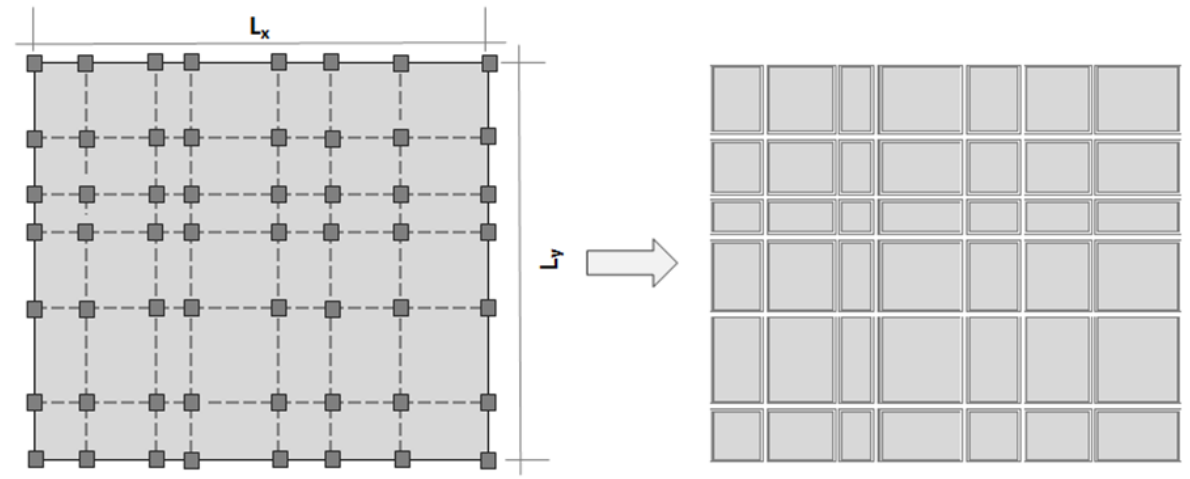

Figure 1: Subdivided Rectangular Plan

\section{Knapsack Problem for Column Layout Design}

Consider rectangular floor plan as shown in Figure 1, whose length and width, $L_{x}$ and $L_{y}$ and Area $A$, are the predetermined dimensions of the plan in the $x$ and $y$ directions, respectively $\left(L_{y} \geq L_{x}\right)$. The aim is to find the optimum column grid layout with maximum profit.

Assume that there is a set of rectangles of various lengths and widths which meet the geometric requirements, such as maximum and minimum spans lengths. These rectangles represent the relative location of columns on their corners and the floor (or slab) surrounded by them. Rectangles are used to carpet a floor plan of area $A$. The problem changes to selecting a set and an appropriate arrangement of rectangles of total area $A$ and an appropriate arrangement of them that maximize the profit (objective function) and meet the constraints. Some constraints must be set to turn the optimum column layout problem to a knapsack problem: The rectangles are placed on the floor plan with their four sides parallel to the $x$ and $y$ axes. That is, each edge of a rectangle is parallel to an edge of the enclosing rectangle. There is also no overlap for any two rectangles. To have all the columns on a grid pattern, the edges shared by any two adjacent rectangles have the same length. This condition prevents the layout plan from off centre columns, and places all the columns on a grid pattern as shown if Fig. 1. Furthermore, there can be no column on the rectangles' edges. Columns only are allowed to be located on corners.

As mentioned above the aim is to determine the number and lengths of spans for each direction. This problem can be treated as a simple knapsack problem as follows:

Having the minimum and maximum allowable span lengths along with the desirable accuracy, a set of rectangles is formed by discretizing the domain spans. This set, as 
the set of items, contains the rectangles of all allowable dimensions, whose lengths and widths represent the spans' lengths in the $y$ and $x$ directions, respectively.

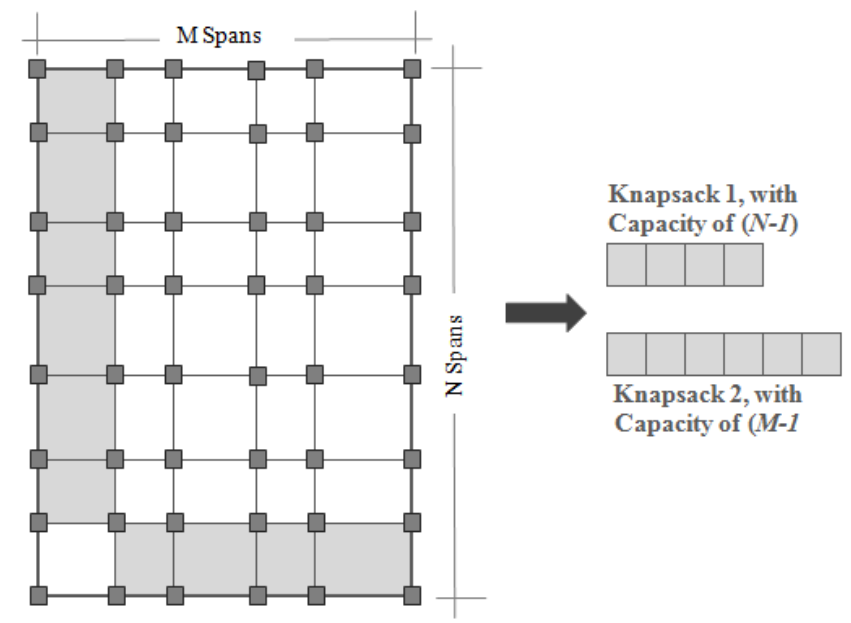

Figure 2: The knapsack model for column layout optimization

Two knapsack of capacities $a_{1}=M-1$ and $a_{2}=N-1$ are set such that the first one contains $M-1$ rectangles on the $x$ axis and the second one contains $N-1$ rectangles on the $y$ axis. The lengths of rectangles in the first knapsack represent the lengths of Spans 2 through $M$ in the $x$ direction, and their width represents the length of Span 1 in the $y$ direction. While, the lengths of rectangles in the second knapsack represent the lengths of Spans 2 through $N$ in the $y$ direction, and their width represents the length of Span 1 in the $x$ direction.

Defining the objective, having the set of items and two knapsacks, the problem of layout optimization of rectangular frames can be formulated by Eqs. (1) through (3), as a multiple knapsack (bi-knapsack) problem. Having the optimum set of rectangles that maximize the profits of the knapsack, the optimum spans in the $x$ and $y$ directions can be determined. In addition to the aforementioned constraints, in case of some other architectural or structural preferences, the constraints can easily be added to the rectangles' selection criteria.

\section{Numerical Example}

A four-storey RC building with a plan as shown in Fig. 3 is optimized. The live load on intermediate floors is $5.0 \mathrm{kN} / \mathrm{m}^{2}$ and on the roof is $1.5 \mathrm{kN} / \mathrm{m}^{2}$. Dead loads are self-weight and the imposed dead load of $1.5 \mathrm{kN} / \mathrm{m}^{2}$. The average unit price for concrete is assumed to be 54 units $/ \mathrm{m}^{3}$, and $3140 \mathrm{units} / \mathrm{m}^{3}$ for reinforcing steel. The average unit price for formwork is 19 units $/ \mathrm{m}^{2}$. The characteristic strength of the main reinforcement $f_{y}$ is $460 \mathrm{~N} / \mathrm{mm}^{2}$, the characteristic strength of the shear reinforcement $f_{y v}$ is $250 \mathrm{~N} / \mathrm{mm}^{2}$, the characteristic strength of concrete $f_{c}^{\prime}$ is 35 
$\mathrm{N} / \mathrm{mm}^{2}$; the top and bottom covers of steel bars are 20 and $25 \mathrm{~mm}$ for slabs, respectively and the cover of bars in columns is $40 \mathrm{~mm}$.

This example was analyzed in a report on comparative costs of concrete framed buildings [7] that has been recommended as a benchmark for future studies, and has been optimized in [6]. The conventional design of this example has been carried out and optimized by a team of professional structural engineers. The total length and width of the building are $20 \mathrm{~m}$ and $18 \mathrm{~m}$ respectively, and the height of each storey is $2.95 \mathrm{~m}$. The permissible spans are defined within the bounds of $L_{\max }=8.5 \mathrm{~m}$ and $L_{\min }=5 \mathrm{~m}$. Goodchild et al. [7] suggest the cost of 239575 units for $L_{x 1}=L_{x 2}=L_{x 3}=$ $L_{x 4}=5.0 \mathrm{~m}$ and $L_{y 1}=L_{y 2}=L_{y 3}=6.0 \mathrm{~m}$. The structural optimization problem has been formulated in detail in [6].

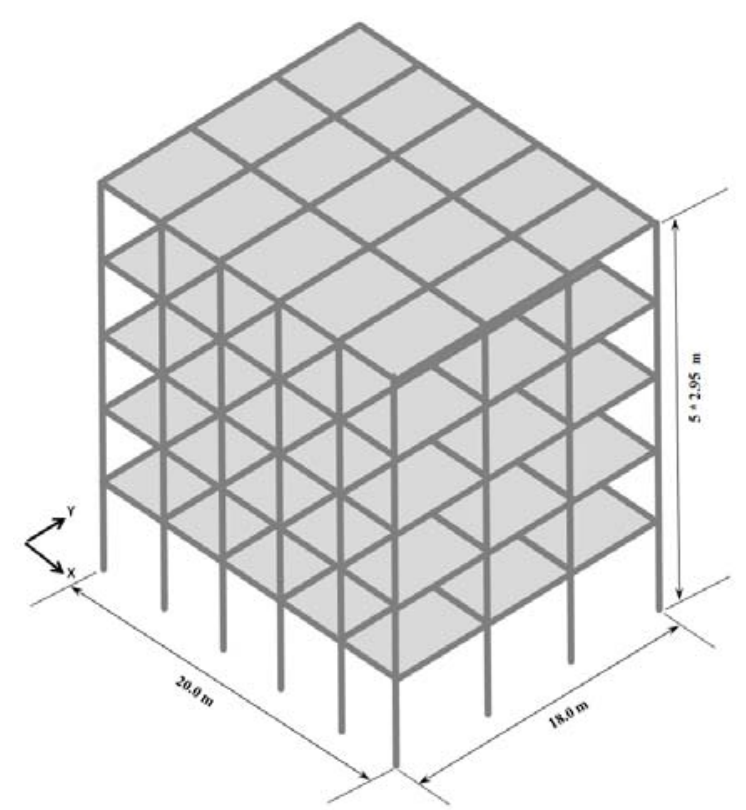

Figure 2: Numerical example, a four story building [6]

Considering the building envelope dimensions and the limitations on the spans lengths, 2 knapsacks of capacities 4 and 2 items are formed for the $\mathrm{y}$ and $\mathrm{x}$ directions respectively to represent the problem ( $m=4, n=2)$. Accuracy of $\varepsilon=0.22 \mathrm{~m}$, results in 15 possible options for rectangles dimensions, which is equivalent to a set of 225 items (rectangles). The characteristics of knapsacks are: Knapsack 1, of capacity 4, whose lengths, representing the lengths of Spans 2 through 5 in the $x$ direction, and width representing the length of Span 1 in the $y$ direction. Knapsack 2, whose lengths representing the lengths of Spans 2 and 3 in the $y$ direction, and width representing the length of Span 1 in the $x$ direction. Therefore the problem of optimum rectilinear shape of the building layout turns to multiple knapsack problem of four rectangles for knapsack 1 and 2 for knapsack 2 from the set of items, such that the total area of rectangles equals $360 \mathrm{~m}^{2}$ and the profit is a maximum. 
Using an Ant colony Optimization (ACO) algorithm, in [6], Sharafi et al. have obtained the optimum span lengths of $L_{x 1}=4550 \mathrm{~mm}, L_{x 2}=5450 \mathrm{~mm}, L_{x 3}=5450$ $\mathrm{mm}$ and $L_{x 4}=4550 \mathrm{~mm}$ in the x direction, and $L_{y 1}=5850 \mathrm{~mm}, L_{y 2}=6300 \mathrm{~mm}$ and $L_{y 3}=5850 \mathrm{~mm}$ in the $y$ direction are obtained, resulting in a total cost of 217534 units, which equals $9.2 \%$ cost saving compared to the initial design.

\section{Concluding Remarks}

An automated combinatorial method is presented for preliminary layout optimization of rectangular framed buildings in which the number and size of spans and the shape of the plan can be considered as variables. To that end, knapsack problem as an applied combinatorial optimization problem is employed. The objective is to find layouts of maximum profit for frames that also satisfy the imposed dimensional constraints.

\section{References}

1. B. Korte, J. Vygen, Combinatorial Optimization: Theory and Algorithms: Springer. 2012,

2. E.L. Lawler, Combinatorial Optimization: Networks and Matroids: Dover Publications. 2001,

3. K.H. Rosen, J.G. Michaels, Handbook of Discrete and Combinatorial Mathematics: CRC Press. 2000,

4. H. Kellerer, U. Pferschy, D. Pisinger, Knapsack Problems: Springer. 2004,

5. P. Sharafi, M.N.S. Hadi, L.H. Teh, Heuristic Approach for Optimum Cost and Layout Design of 3D Reinforced Concrete Frames. Journal of Structural Engineering, 138(7): p. 853-863. 2012.

6. P. Sharafi, M.N.S. Hadi, L.H. Teh, Optimum Conceptual Design of Frames of Rectilinear Patterns: Algorithm and Applications for Reinforced Concrete Buildings Journal of Structural Engineering, (submitted). 2013.

7. C.H. Goodchild, R.M. Webster, K.S. Elliott, Economic Concrete Frame Elements to Eurocode 2: A Pre-scheme Handbook for the Rapid Sizing and Selection of Reinforced Concrete Frame Elements in Multi-storey Buildings Designed to Eurocode 2. UK: The Concrete Center. Michael Burbridge Ltd, Maidenhead. 2009, 